\title{
An Efficient Edge Server Selection in Content Delivery Network using Dijkstra's Shortest Path Routing Algorithm with Euclidean Distance
}

\author{
Sougata Chakraborty \\ Department of Computer Science \& Engineering \\ University of Kalyani \\ Kalyani, India
}

\author{
Debabrata Sarddar, Ph.D \\ Department of Computer Science \& Engineering \\ University of Kalyani \\ Kalyani, India
}

\begin{abstract}
The performance enhancement of the edge server in Content Delivery Network is the budding area of the research in the fields of modern Information Technology and Communication Engineering so that the web users or the cloud users across the network get faster service irrespective of any geographic locations during browsing over internet and at the same time downloading multimedia contents. As an aftermath, several approaches and aspects have been introduced so far with the efflux of time. Therefore, our main objective is to facilitate this area of improvement by finding the selection of an appropriate edge server which will provide the multimedia contents and ensuring a faster response time and downloading time of the requested content due to reduced latency to the web users as well. Our proposed method will show the efficient technique for choosing the appropriate edge server with comparatively less complexity.
\end{abstract}

\section{Keywords}

Edge Server; Cloud user; Content Delivery Network; Shortest Path Routing Algorithm; Nearest Neighbor Edge Server;

\section{INTRODUCTION}

In the modern era of Information Technology, the internet service is evolving day by day. With the rapid progress of internet, the question comes of the scalability and speed up factor. Most illustratively speaking, the performance issue of a network could be the major factor in the global perspective. As a consequence, the concept of content distribution over the internet becomes the relevant point.

\section{BACKGROUND STUDY}

\subsection{Preliminaries}

Once upon a time, remote access and file transfer were the two prime objectives to the user which were introduced as telnet and ftp respectively [6]. Later, those protocols were used inevitably in the giant organizations' intra-network. Over the years, many software have been launched by the several organizations to serve the same purpose and used in the global internetwork as well. Since the last decade, the major application of the file transfer protocol has become web hosting. Similarly, another aspect of the file transfer mechanism is file sharing through email and messenger service. In today's world, with the simultaneous use of the previous services, the trend of downloading multimedia contents have come into the picture. In this context, reliability and consistency of the data are the two major impacting concerns naturally. Henceforth, the client-server model is introduced where a client process sends a request to a server process for getting some service. The server executes the request and sends back a reply to the client that contains the result of request processing. This model provides an effective general-purpose approach to the sharing of information and resources in distributed computing systems.

\subsection{Problems and Approaches}

With the explosive Web growth in this modern era, scalability and speed-up have become the most critical challenges to the Internet [6]. Now a days, a popular web server has to serve a huge number of web users in a short span of time. At the same time, multiple requests coming to a web server which can overload the web server and its accessible links. As a result, links get failure in the business hours and this phenomenon hampers our daily important online activities.

To overcome this hurdle, the new approach, called 'content distribution', has been developed.

\section{CONTENT DELIVERY NETWORK}

A Content Delivery Network or Content Distribution Network $(\mathrm{CDN})$ is basically a large distributed system of servers across the global internet. The edge servers are used to deliver the web contents to the requested users based on their geographic locations [1], [2], and [4]. But the average response time of the original host server of any popular website requested by the users becomes very higher. Solution to this problem is the selection of the edge servers which are geographically dispersed using efficient routing algorithm. When a user requests for web content the $\mathrm{CDN}$ redirects the user's request from the original host server to an edge server that is closest to the requested user. This technique also helps to reduce the average response time and improves the average packet loss of the CDN [3]. In this paper we have specially found out an approach to the selection of edge servers using Dijkstra's Shortest Path Routing algorithm with Euclidean Distance.

A Content Delivery Network (CDN) is a distributed network of globally dispersed edge servers which caches the static and some dynamic content of a webpage like the flash, images, audios, videos, Ajax, cascading Style Sheet (CSS) and Java Script (JS) or other users' related files [4]. When users from different geographical locations request a website then the nearest edge server to the user will deliver that static and dynamic content with the help of CDN and reduce the network latency time.

\section{DIJKSTRA'S SHORTEST PATH ROUTING ALGORITHM}

Djikstra's algorithm finds the shortest path from a point in a graph (the source) to a destination. One can find the shortest paths from a given source (with respect to any node) to all the points in a graph and prior to invention of Djikstra's algorithm, this problem was sometimes called the singlesource shortest path problem. 


\section{EUCLIDEAN DISTANCE}

The Euclidean distance between points $p$ and $q$ is the length of the line segment connecting them $(p q)$

$$
d(p q)=\sqrt{\sum_{i=1}^{n}\left(q_{i}-p_{i}\right)^{2}}
$$

\section{PROPOSED METHOD}

In our work, we have specially found out an approach to the selection of an appropriate edge server to deliver web contents to the requested users based on their geographic locations with the reduction of the average response time as well as the improvement of the average packet loss in deed.

Here the set of edge servers are considered as the set of nodes $=\{\mathrm{C}, \mathrm{D}, \mathrm{E}, \mathrm{F}, \mathrm{G}, \mathrm{H}\}$ scattered over the geographical region.

Here we have considered the edge servers located in six different locations across the globe.

Executing 'ping' command over all the neighbor edge server's IP address, we can simply capture the accurate network latency time. Now, the minimum network latency time can be obtained easily from the captured result set.

Now, we can assume each and every network latency time as the weights of the constituent edges. Thus, we can easily calculate the minimum length path by using Dijkstra's Shortest Path Algorithm.

1)

However, we can get the shortest path with respect to the associated servers. The following figure Fig 1 shows the pictorial example of the obtained shortest path using Dijkstra's Algorithm.

Here we have obtained the best shortest path from $(\mathrm{C})$ to $(\mathrm{H})$, which is $(\mathrm{C})-(\mathrm{E})-(\mathrm{F})-(\mathrm{H})$ with cost 5 .

Let the set of nodes (C)-(D)-(E)-(F)-(G)-(H) are considered as the set of points $P$ scattered over the globe. Similarly, a group of requested users are considered as the set of points $\mathrm{Q}$ located in the different locations. We can simply find the Euclidean distance between each P and each Q.

If we compare each and every computed distance value with respect to a particular node, then by taking minimum value, we can simply find out that which node could be the nearest neighbor edge server of any requested user.

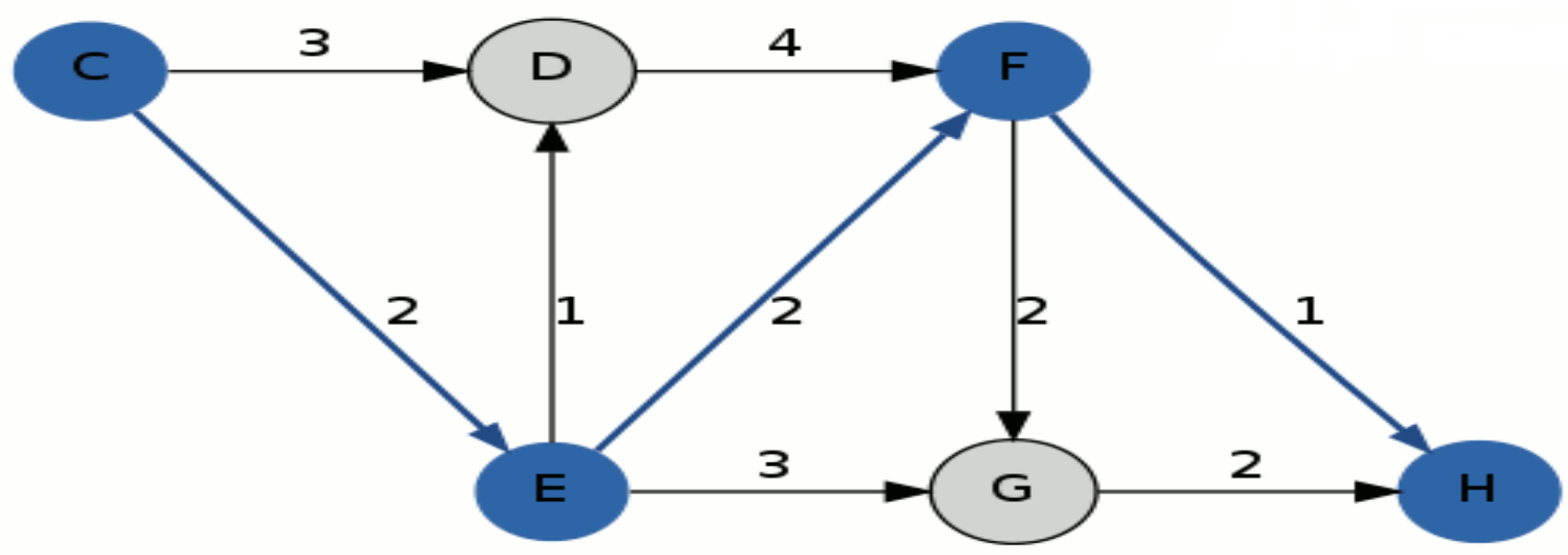

Fig 1: Dijkstra's Shortest Path

\section{RESULTS}

Table 1. Selection of the appropriate edge server using the minimum Euclidean Distance value with respect to a particular requested user

\begin{tabular}{|l|l|l|l|l|}
\hline $\begin{array}{l}\text { Requested } \\
\text { User }(\mathbf{Q})\end{array}$ & $\begin{array}{l}\text { Co- } \\
\text { ordinate } \\
\text { point of } \\
\text { any } \\
\text { Requested } \\
\text { User } \\
\left(x_{1}, y_{1}\right)\end{array}$ & $\begin{array}{l}\text { Edge } \\
\text { Servers } \\
(\mathbf{P})\end{array}$ & $\begin{array}{l}\text { Co- } \\
\text { ordinate } \\
\text { points of } \\
\text { the Edge } \\
\text { Servers } \\
\left(x_{2}, y_{2}\right)\end{array}$ & $\begin{array}{l}\text { Euclidean } \\
\text { Distance } \\
(d)\end{array}$ \\
\hline$q_{1}$ & $(1,1)$ & $\mathrm{C}$ & $(1,2)$ & 1 \\
\hline & & $\mathrm{D}$ & $(1,4)$ & 3 \\
\hline & & $\mathrm{E}$ & $(1,3)$ & 2 \\
\hline & & $\mathrm{F}$ & $(1,5)$ & 4 \\
\hline & $\mathrm{G}$ & $(1,6)$ & 5 \\
\hline
\end{tabular}

\section{CONCLUSION}

Our main focus was finding an approach to the selection of edge servers with less complexity so that the web users or the cloud users might get faster service during browsing over internet and downloading multimedia contents from any popular website if the contents come from the nearest neighbor edge server and traverse along the shortest path. This method can be used in irrespective of any wired or wireless network and even mobile or cloud network also. Further studies are necessary to get the optimized result using efficient clustering algorithm for a large network in order to reach the apex Quality of Service and performance benchmark. Thus, a new algorithm might be developed as well as devised for the sake of mankind.

\section{ACKNOWLEDGMENTS}

I would like to convey my profound respect and sincerest gratitude to my reverend research advisor Asst. Prof. Dr. Debabrata Sarddar for sparing his precious time and integrating new flawless ideas at every stage of our work. Without his spontaneous kind co-operation, motivation, and meticulous guidance, I would not have been able to carry out this research work successfully. 


\section{REFERENCES}

[1] E. Nygren, R. K. Sitaraman and J. Sun. Article: The Akamai Network: A Platform for High-Performance Internet Applications. ACM SIGOPSOSR, vol. 44, no. 3, pp. 2-19, 2010.

[2] T. Repantis, J. Cohen, S. Smith and J. Wein. Article: Scaling a Monitoring Infrastructure for the Akamai Network. ACM SIGOPS Operating Systems Review, vol. 44, no. 3, July 2010.

[3] J. Parikh, H. Prokop, R. Sitaraman, J. Dilley, B. Maggs and B. Weihl. Article: Globally Distributed Content Delivery. IEEE INTERNET COMPUTING, pp. 50-58, September-October 2002.

[4] Debabrata Sarddar, Sandip Roy and Rajesh Bose. Article: An Efficient Edge Servers Selection in Content Delivery Network Using Voronoi Diagram. International Journal on Recent and Innovation Trends in Computing and Communication Volume: 2 Issue: 8. ISSN: 23218169.

[5] Jun Li, Snigdha Verma and Kumar Ramaswamy. Patent: Centralized scheduler for content delivery network. Link: http://www.google.co.in/patents/WO2007111588A1?cl= en Publication Number: WO2007111588 A1.

[6] J. Pan et al. Article: An overview of DNS-based server selections in content distribution networks. Elsevier Computer Networks 43 (2003) 695-711.

[7] P. Danzig, K. Obraczka and A. Kumar. Article: An analysis of wide area name server traffic: a study of the Internet domain name system. In: Proc. ACM SIGCOMM'92, 1992, pp. 281-292.
[8] K. Johnson, J. Carr, M. Day and M. Kaashoek. Article: The measured performance of content distribution networks. In: Proc. IEEE WCW'01, Boston, MA, 2001.

[9] B. Krishnamurthy, C. Wills and Y. Zhang. Article: On the use and performance of content distribution networks. In: Proc. 1st ACM IGCOMM Internet Measurement Workshop (IMW'01), San Francisco, CA, 2001.

[10] A.Shaikh, R. Tewari and M. Agrawal. Article: On the effectiveness of DNS-based server selection. In: Proc. IEEE INFOCOM'01, 2001, pp. 1801-1810.

[11] Debabrata Sarddar, Sandip Roy and Rajesh Bose. Article: A Tool based Edge Server Selection Technique using Spatial Data Structure. Global Journal of Computer Science and Technology: G Interdisciplinary. Volume 14 Issue 3 Version 1.0 Year 2014. Type: Double Blind Peer Reviewed International Research Journal. Publisher: Global Journals Inc. (USA). Online ISSN: 0975-4172 \& Print ISSN: 0975-4350.

[12] Debabrata Sarddar, Sandip Roy and Rajesh Bose. Article: Queueing Based Edge Server Selection in Content Delivery Network Using Haversine Distance. International Journal of Electronics Communication and Computer Technology (IJECCT). Volume 4 Issue 5 (September 2014). ISSN: 2249-7838.

[13] P. Christy. Article: The network provider's business case for Internet content delivery. Internet Research Group. Link: http://www.irgintl.com/ , 1999. 